\title{
Mosquito repellents for travellers
}

\author{
Nina M Stanczyk research fellow ${ }^{1}$, Ron H Behrens senior lecturer ${ }^{12}$, Vanessa Chen-Hussey research \\ scientist and clinical trials manager ${ }^{13}$, Sophie A Stewart senior research scientist and clinical trials \\ coordinator $^{3}$, James G Logan senior lecturer ${ }^{13}$
}

${ }^{1}$ Department of Disease Control, London School of Hygiene and Tropical Medicine, London WC1E 7HT, UK; ${ }^{2}$ Hospital for Tropical Diseases, London, UK; ${ }^{3}$ Arthropod Control Product Test Centre, London School of Hygiene and Tropical Medicine, London, UK

\begin{abstract}
This is one of a series of occasional articles on therapeutics for common or serious conditions, covering new drugs and old drugs with important new indications or concerns. The series advisers are Robin Ferner, honorary professor of clinical pharmacology, University of Birmingham and Birmingham City Hospital, and Albert Ferro, professor of cardiovascular clinical pharmacology, King's College London. To suggest a topic, please email us at practice@bmj.com.
\end{abstract}

\section{Case scenario}

A pregnant woman visits you as her general practitioner (GP) because she and her children will be visiting a country with mosquito borne disease. You recommend using repellents to protect against mosquitoes, as well as vaccinations and other relevant disease prevention measures. She asks which repellents would be best.

\section{What are the active ingredients?}

The key factors to consider when choosing a repellent are the active chemical ingredients and the strength (concentration (\%) of active ingredient) because these influence the efficacy and duration of protection. ${ }^{12}$ There are four active ingredients with sufficient published scientific evidence to warrant recommendation. Repellents are useful in areas of low risk of mosquito borne disease to prevent nuisance biting (which may lead to problems such as allergies) and are essential in moderate to high risk areas (figure $\Downarrow$ ) to prevent disease transmission (such as malaria and dengue fever) through bites. Repellents work on mosquitoes by directly stimulating avoidance behaviour or by blocking the mosquito's receptors for attractive odours, not though toxicity. ${ }^{3}$

DEET - $N, N$-Diethyl-meta-toluamide has been in use since 1946 and is the "gold standard" repellent recommended by the World Health Organization Pesticide Evaluation Scheme. ${ }^{4}$

PMD-Many people prefer the idea of a "natural" repellent to a synthetic one. $p$-Menthane-3,8-diol (PMD) was first isolated as a byproduct of Eucalyptus citriodora (lemon eucalyptus).

Icaridin-Icaridin (hydroxyethyl isobutyl piperidine carboxylate) is also known by the trade names Bayrepel, Picaridin, and Saltidin.

Insect Repellent 3535-IR3535 (ethyl

butylacetylaminopropionate) is a synthetic repellent that has been less comprehensively studied.

\section{How well do repellents work?}

Although this article focuses on mosquitoes, the four recommended active ingredients may also protect against other arthropod vectors, such as sand flies and ticks. The recommended active ingredients should repel up to $100 \%$ of mosquitoes of the genera Aedes, Anopheles, and Culex for a specified duration. Although some repellents can last up to 12 hours, the average is 4-8 hours, depending on the active agent, application type, and the local mosquito species. ${ }^{2}$

DEET-For disease endemic countries DEET should be present at 20-50\% concentration, because several large well conducted randomised control trials have shown that this concentration offers complete protection by repelling $100 \%$ of Aedes, Culex, and Anopheles mosquitoes for 6-13 hours. ${ }^{12}$

PMD_Repellents with 30\% PMD provided complete protection for 4-6 hours against Aedes, Culex, and Anopheles mosquitoes in randomised controlled trials. ${ }^{12}$ Apart from its shorter duration of action, efficacy is similar to DEET.

Icaridin-Like DEET, in randomised controlled trials in the field, at concentrations greater than $20 \%$, Icaridin offered complete protection for up to six hours against Anopheles, Aedes, and Culex species. ${ }^{1}$

IR3535-This has shown complete protection, comparable to DEET, at $20 \%$ concentration against several mosquito species including Aedes and Culex for 7-10 hours in well 


\section{The bottom line}

- Always recommend a topically applied repellent with a proven active ingredient such as DEET (20-50\%), PMD (30\%), or Icaridin $(20-50 \%)$. IR3535 (20\%) is recommended only for areas that are not malaria endemic

- Reapply repellents at least every six to eight hours if using DEET or IR3535, or every four to six hours for PMD and Icaridin, and sooner if they wear off while swimming or sweating in warm weather

- DEET can be used on children over 8 weeks old, PMD on children over 3 years

DEET is safe for use from the second trimester onwards and while breast feeding

replicated laboratory longevity studies and non-randomised field trials in Thailand. However, protection time is shorter $(\sim 3 \mathrm{~h})$ against Anopheles species, so it should not be recommended for malaria endemic regions.

\section{How safe are repellents?}

During the 1980s and 1990s there were several reports of encephalopathy following DEET exposure in children. ${ }^{1}$ However, risk assessments by both the US Environmental Protection Agency (USEPA) and independent publications, as well as a clinical trial, found no association between encephalopathy and DEET use, and no toxological risk or severe effects except after inappropriate use (ingestion, direct inhalation, or eye exposure). ${ }^{5}$ PMD, Icaridin, and IR3535 have also been registered for safe use as repellents by the USEPA ${ }^{6}$ and $\mathrm{WHO},{ }^{78}$ but have not been extensively studied in humans.

\section{What are the precautions?}

Pregnancy and breast feeding-No trials have assessed the safety of PMD, Icaridin, or IR3535 in human pregnancy or during breast feeding, so these drugs cannot be recommended. A large, well conducted, double blind randomised controlled trial (RCT) in Thailand of 897 pregnant women showed no adverse effects of topically applied DEET in women or their infants when followed for one year after birth, including while breast feeding. ${ }^{9}$ Participants were all in the second or third trimester, however, and no human trials provide safety data for earlier in pregnancy. Nonetheless, studies in rats and rabbits showed no effects on offspring, suggesting that DEET is safe when used as recommended. ${ }^{10}$

Children-Topical repellents are recommended for children of all ages unless the label specifically states an age restriction. ${ }^{11}$ Products containing DEET are considered safe for use in children over 2 months of age. ${ }^{10}$ Products containing PMD are not advised in children under 3 years because of the lack of safety data for this age range. ${ }^{11}$ The repellent should be applied by an adult who has thoroughly read the directions. A third to a half of parents apply repellent incorrectly to children - for example, by applying it to the children's clothing as well as their skin or by not removing it before putting children to bed. ${ }^{12}$

Sunscreen-Limited early laboratory research suggested that applying 33.5\% DEET after sunscreen significantly reduces the sun protection factor (SPF) of the sunscreen. ${ }^{13}$ Randomised controlled trials with Aedes and Anopheles mosquitoes found that applying repellent concurrently with or before sunscreen did not lessen the effect of the repellent, ${ }^{14}{ }^{15}$ but that reapplication of the sunscreen over the repellent reduced the mean repellent protection time by one hour. ${ }^{15}$ Thus advise travellers to apply repellent first or use a combined repellent and sunscreen product and be aware that repellent may wear off more quickly if reapplying only sunscreen on top. If using a combination product, ensure that the concentration of repellent is sufficient.

\section{How are they used?}

Recommend topical repellents, such as lotions and sprays, because they are the most studied and have the greatest efficacy. ${ }^{1}$ The repellent should be effective against the vector species present at the destination. Thus, although all four repellents are effective, do not recommend IR3535 for malaria endemic regions, given its shorter duration of action against Anopheles spp. Apply evenly to all exposed skin except the face (to avoid accidental eye exposure or ingestion) during times of risk (at dawn, dusk, and evening for most regions, but also during the day, particularly in South East Asia, South America, and in forested areas). Although every product is different, as a general rule, re-apply DEET and IR3535 every six to eight hours, and PMD and Icaridin every four to six hours. ${ }^{12}$ The tips for travellers box provides more detail on appropriate use.

\section{How do they compare with alternatives?}

An RCT compared common commercial products containing essential oils, vitamin $B_{1}$, or the insecticide metofluthrin, in different delivery systems, including wristbands, stickers, patches, sonic devices, and diffusers. ${ }^{16}$ It found that only the personal diffusers containing metofluthrin or a mix of essential oils had any repellent effect, reducing localised biting by $87-95 \%$. There is no evidence that any of these devices provide adequate protection for areas with mosquito borne disease.

Other active ingredients include essential oils such as citronella, neem, thyme, geraniol, peppermint, patchouli, and clove. Because these compounds are volatile, efficacy is variable. They may provide $20-100 \%$ protection for about two hours, but a recent systematic review of laboratory and field trials found no evidence that they can protect against disease transmission. ${ }^{17}$

There is anecdotal evidence that a change in diet and vitamin supplements can protect against mosquito bites. Although this has not been looked at extensively, an RCT of the effect of vitamin $B_{12}$ on human odour showed that it has no effect on mosquito biting rates. ${ }^{18} \mathrm{~A}$ double blinded RCT that tested the effect of garlic supplements as mosquito repellents found no protective effect. ${ }^{19}$ Intake of supplements like vitamin B or garlic should not be recommended for protection against mosquitoes.

\section{Outcome}

You recommend a topical repellent with DEET (20-50\%) because it is effective and safe for pregnant women and children and provide advice on how to apply it (see tips for travellers box).

Contributors: All authors helped design and plan the manuscript; NMS, VC-H, SAS, and JGL co-wrote the manuscript; and RHB critically revised it. All authors were involved in subsequent revisions. JGL is guarantor. 


\section{Tips for travellers}

Use a repellent containing $20-50 \%$ DEET or Icaridin, or 30\% PMD. Use IR3535 (20\%) only if travelling to a country without malaria. Higher concentrations of DEET will repel mosquitoes for longer than lower concentrations. However, products with greater than $50 \%$ DEET are not recommended

Essential oils such as citronella, repellent wristbands, garlic supplements, and vitamin $\mathrm{B}_{12}$ do not provide adequate protection against biting and disease transmission

Apply repellent evenly to all exposed skin except the face, particularly when there are lots of mosquitoes, such as in the early morning and the evening, but also during the day if mosquitoes are present

DEET and IR3535 should be reapplied every six to eight hours, and PMD and Icaridin every four to six hours, unless stated otherwise on the label. If swimming or sweating in warm weather, they may wear off sooner and will need to be reapplied

Apply repellent and sunscreen simultaneously or repellent first; if using a combination product, check that it contains the right concentration of repellent $(20-50 \%$ DEET)

You can safely use DEET when pregnant (from the second trimester onwards), when breast feeding, and on children over 2 months old. Thoroughly cover the child's exposed skin (except the face), not clothing, and ensure that you wash it off before bed Additional resources: www2.epa.gov/insect-repellents; wwwnc.cdc.gov/travel/page/avoid-bug-bites

Competing interests: We have read and understood BMJ policy on declaration on interests and declare the following interests: JGL, VC-H, and SAS are employees of the Arthropod Control Product Test Centre, a wholly owned subsidiary of the London School of Hygiene and Tropical Medicine that performs independent efficacy testing of repellents for commercial companies. The other authors have no competing interests. Provenance and peer review: Not commissioned; externally peer reviewed.

Patient consent not required (patient anonymised, dead, or hypothetical).

1 Lupi E, Hatz C, Schlagenhauf $P$. The efficacy of repellents against Aedes, Anopheles, Culex and Ixodes spp-a literature review. Travel Med Infect Dis 2013;11:374-411.

2 Goodyer LI, Croft AM, Frances SP, Hill N, Moore SJ, Onyango SP, et al. Expert review of the evidence base for arthropod bite avoidance. J Travel Med 2010;17:182-92.

3 Bohbot JD, Dickens JC. Insect repellents: modulators of mosquito odorant receptor activity. PloS One 2010; doi:10.1371/journal.pone.00121385(8).

4 WHO. Guidelines for efficacy testing of mosquito repellents for human skin. 2009. http:// whqlibdoc.who.int/hq/2009/WHO_HTM_NTD_WHOPES_2009.4_eng.pdf.

5 Chen-Hussey V, Behrens R, Logan JG. Assessment of methods used to determine the safety of the topical insect repellent N,N-diethyl-m-toluamide (DEET). Parasit Vectors 2014;7:173.

6 United States Environmental Protection Agency. Science review in support of the registration of Claire-1 containing p-menthane-diol. 2004. www.epa.gov/opp00001/chem search/cleared_reviews/csr_PC-011550_20-Jan-04_a.pdf.

7 WHO. Icaridin. WHO specifications and evaluations for public health pesticides. 2004 www.who.int/whopes/quality/en/lcaridin_spec_eval_Oct_2004.pdf.
8 WHO. Ethyl butylacetylaminopropionate (IR3535). WHO specifications and evaluations for public health pesticides. 2006. www.who.int/whopes/quality/en/IR3535 eval april 2006.pdf.

9 McGready R, Hamilton KA, Simpson JA, Cho T, Luxemburger C, Edwards R, et al. Safety of the insect repellent N,N-diethyl-M-toluamide (DEET) in pregnancy. Am J Trop Med Hygiene 2001;65:285-9.

10 Koren G, Matsui D, Bailey B. DEET-based insect repellents: safety implications for children and pregnant and lactating women. CMAJ 2003;169:209-12.

11 US Food and Drug Administration. Insect repellent use and safety in children. 2012. www. fda.gov/Drugs/EmergencyPreparedness/ucm085277.htm.

12 Menon KS, Brown AE. Exposure of children to DEET and other topically applied insect repellents. Am J Industr Med 2005;47:91-7.

13 Montemarano AD, Gupta RK, Burge JR, Klein K. Insect repellents and the efficacy of sunscreens. Lancet 1997;349:1670-1.

14 Murphy ME, Montemarano AD, Debboun M, Gupta R. The effect of sunscreen on the efficacy of insect repellent: a clinical trial. J Am Acad Dermatol 2000;43(2 Pt 1):219-22.

15 Webb CE, Russell RC. Insect repellents and sunscreen: implications for personal protection strategies against mosquito-borne disease. Aust N Z J Public Health 2009;33:485-90.

16 Revay EE, Junnila A, Xue RD, Kline DL, Bernier UR, Kravchenko VD, et al. Evaluation of commercial products for personal protection against mosquitoes. Acta Trop 2013;125:226-30.

17 Maia MF, Moore SJ. Plant-based insect repellents: a review of their efficacy, development and testing. Malaria J 2011;10(suppl 1):S11.

18 Ives AR, Paskewitz SM. Testing vitamin B as a home remedy against mosquitoes. J Am Mosquito Control Assoc 2005;21:213-7.

19 Rajan TV, Hein M, Porte P, Wikel S. A double-blinded, placebo-controlled trial of garlic as a mosquito repellant: a preliminary study. Med Vet Entomol 2005;19:84-9.

Accepted: 23 December 2014

Cite this as: BMJ 2015;350:h99

(c) BMJ Publishing Group Ltd 2015 


\section{Figure}

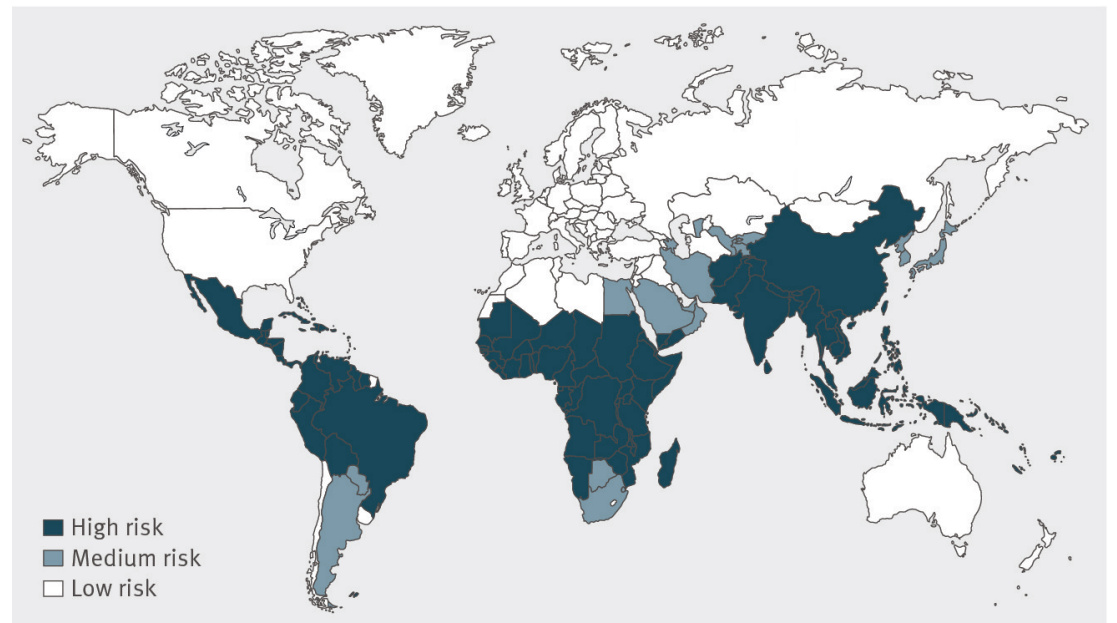

Areas of low, moderate, and high risk of mosquito borne disease worldwide 